\title{
Subjetividad juvenil: lecturas desde y para el Trabajo Social en Colombia
}

\section{Daniela Joya-Valbuena}

Magíster en Hábitat. Trabajadora Social

Universidad Nacional de Colombia. Bogotá, Colombia

https:/ / orcid.org/0000-0001-8573-1715・djoyav@unal.edu.co

Resumen

Este artículo presenta las reflexiones derivadas de la revisión de la producción académica del Trabajo Social colombiano en torno a la subjetividad juvenil y el quehacer profesional respecto a dichas subjetividades. Se basa en los hallazgos de un estado del arte en el cual se revisaron documentos escritos en revistas indexadas de Trabajo Social y en otras revistas de Ciencias Sociales que dentro de sus reflexiones e investigaciones abordaron, con una mirada crítica, la construcción cultural e histórica del joven como un sujeto subalternizado en una sociedad adultocéntrica. En este sentido, se analiza la arqueología del concepto de juventud en la sociedad occidental, se revisan los discursos dominantes y se resignifica la perspectiva desde las categorías de identidad, subjetividad y resistencia. Así mismo, se revisa el lugar de la profesión-disciplina en esta relación y se plantean retos y desafíos para dar un giro conceptual y epistemológico en la intervención y la investigación.

Palabras clave: Subjetividad; Trabajo Social; Adultocentrismo; Juventud.

Recibido: 10/01/2020 | Aprobado: 05/05/2021 |Publicado: 01/07/2021

(c) (1) Esta obra está bajo una Licencia Creative Commons Atribución-NoComercialCompartirIgual 4.0 Internacional.

Financiación o proveniencia del artículo: Este artículo presenta parte del estado del arte del trabajo monográfico Subjetividad juvenil: lecturas desde y para el Trabajo Social en Colombia realizado en el Departamento de Trabajo Social de la Universidad Nacional de Colombia, Sede Bogotá, en el año 2017.

¿Cómo citar este artículo? / How to quote this article?

Joya-Valbuena, D. (2021). Subjetividad juvenil: lecturas desde y para el Trabajo Social en Colombia. Prospectiva. Revista de Trabajo Social e intervención social, (32), 79-102. doi: 10.25100/prts.v0i32.10919. 
Joya-Valbuena

\title{
Youth Subjectivity: Readings from Within and For Social Work in Colombia
}

\begin{abstract}
This paper presents the considerations that came from a review of the academic production of Colombian Social Work regarding youth subjectivities and the associated professional roles and duties. It is based on the results of a state of the art that reviewed documents, published in indexed journals of Social Work and in other Social Studies journals, which critically approached the issue of the cultural and historical construction of youth as subaltern subjects in a society marked by adult centrism. In this sense, the paper analyzes the archeology of the concept of youth in western societies, reviews the dominant discourses and re-signifies the perspective based on the categories of identity, subjectivity and resistance. Also, it reviews the role of the profession-discipline in this relationship and it proposes challenges to make a conceptual and epistemological shift in intervention as well as in research.
\end{abstract}

Keywords: Subjectivity; Social Work; Adult centrism; Youth.

Sumario: 1. Introducción, 2. Metodología, 3. Hallazgos, 3.1. Revisión histórica de la concepción de juventud en occidente y en Colombia, 3.2 Ser joven en una sociedad adultocéntrica, 3.3. Adaptación, estigmatización y neoliberalismo, 3.4. El "deber ser" del joven y la familia, 3.5. Nuevos abordajes del joven: del sujeto problemático al sujeto con agencia, 3.6. El encuentro intra-generacional como parte de la construcción subjetiva, 3.7 Resignificar la rebeldía desde las prácticas de resistencia y la búsqueda de autonomía, 4. Conclusiones, 5. Agradecimientos, 6. Referencias Bibliográficas. 


\section{Introducción}

El encuentro con jóvenes hace parte de los distintos escenarios de intervención en Trabajo Social tanto a nivel institucional como comunitario y, así mismo, se enmarca dentro de las principales discusiones en la construcción de conocimiento sobre nuestro ser y quehacer profesional y disciplinar. Sin embargo, estas perspectivas no se escapan de los discursos dominantes que permean la institucionalidad y el "deber ser" de los sujetos en la sociedad occidental, la cual ha consolidado formas de relacionamiento hegemónicas alrededor de la generación.

Es por ello, que este artículo busca identificar las principales reflexiones que desde el Trabajo Social colombiano se han desarrollado respecto a la subjetividad juvenil, teniendo en cuenta las apuestas que nuestra profesión-disciplina ha trazado para proponer un giro epistemológico y conceptual que supere la mirada tradicional de "etapa" que subalterniza a las y los jóvenes y en cambio, los reconozca como agentes de transformación tanto en la intervención como en la investigación.

Abordar la juventud desde la categoría de subjetividad permite pensar el ser en relación y desde la complejidad; no es un individuo aislado que se produce a sí mismo de manera independiente, sino que se encuentra en constante interacción y a partir de allí va construyendo sentidos y significados. Ahora bien, este encuentro con los demás no es lineal y armónico sino que implica una serie de tensiones que están atravesadas por elementos simbólicos y materiales (Sierra-Pardo, 2015); ya que la subjetividad está condicionada por relaciones de poder asimétricas que se han naturalizado históricamente y se han legitimado a través de las normas y los valores que las sociedades establecen como deseables.

Los discursos dominantes y los distintos niveles de introyección y apropiación de los mismos hacen parte de la constitución subjetiva; las perspectivas de realidad tienen que ver con un proceso de construcción histórica de valores y normas que hacen que el sujeto sea sujeto en tanto "es sometido a otro a través del control y la dependencia y (...) atado a su propia identidad por la conciencia o el conocimiento de sí mismo" (Foucault, 1988, p. 7). De este modo, el poder y el control se normalizan a través del lenguaje y de las estructuras sociales que dan forma a la política, la economía, la cultura, la familia y la vida comunitaria.

Por otra parte, analizar la subjetividad implica abordar la capacidad de agencia del sujeto frente a su propia vida, sus relaciones y su entorno. La comprensión de la subjetividad desde el poder y la producción histórica de valores está acompañada por la posibilidad de emergencia de subjetividades que resisten y que son capaces de crear otros modos de relacionamiento que cuestionan las estructuras (Foucault, 1988); la potencia de esta categoría está en su carácter dinámico e inacabado, las subjetividades no están dadas sino que se encuentran en constante movimiento. Es por ello que el abordaje desde la 
Joya-Valbuena

subjetividad permite ver a los sujetos no solo como productos sino también como productores capaces de transformar condiciones simbólicas y materiales de las estructuras y del ejercicio del poder en las relaciones cotidianas (Sierra-Pardo, 2015).

Ahora bien, desde la mirada crítica sobre la cual se sitúa esta reflexión, es importante hacer una distinción entre la subjetividad juvenil y el concepto de "adolescencia". Al cuestionar las construcciones simbólicas dominantes, nuestra perspectiva pone en entredicho la existencia de una "etapa" entre los 12 y 17 años que se ha consolidado a partir de lo "problemático" o la "rebeldía" propia de una "transición biológica" de "la infancia a la madurez" (Cajiao, 2008, p. 378). Si bien la jerarquía intergeneracional contiene una separación etaria sobre la que se han difundido características homogéneas para todas las épocas y contextos, estas no deben abordarse de manera acrítica sino que contienen en sí mismas un entramado de sentidos que atraviesan las identidades y permean la cultura. Consideramos entonces, que la noción de "adolescencia" subalterniza y legitima las concepciones negativas asociadas a la juventud.

Con estas precisiones sobre el horizonte que sustenta nuestra mirada, planteamos las principales discusiones que se han dado desde el Trabajo Social colombiano y que abren múltiples caminos para la transformación de las lógicas que permean nuestro ser y nuestro quehacer. Estas reflexiones se basan en un estado del arte elaborado por la autora, en el cual se tuvo en cuenta la producción académica sobre la subjetividad juvenil, desarrollada por nuestra profesión-disciplina en el periodo 1996-2016. Para ello, se llevó a cabo una búsqueda y análisis de artículos publicados en revistas indexadas de Trabajo Social colombiano y se tuvieron en cuenta también documentos de trabajadores y trabajadoras sociales que escribieron al respecto en otras revistas de Ciencias Sociales del país durante este periodo.

En un primer momento, el artículo presenta la metodología que sustentó y permitió la consolidación de los hallazgos derivados de la revisión de literatura los cuales parten de la construcción histórica de la concepción dominante de juventud en Occidente y en Colombia, para luego situar la reflexión sobre la sociedad adultocéntrica y neoliberal que determina un "deber ser" para las y los jóvenes. Posteriormente se plantea la discusión sobre el concepto de subjetividad juvenil como un nuevo abordaje posible, teniendo en cuenta las interacciones, las distintas tensiones de la vida cotidiana y la emergencia de distintas formas de resistencia.

A partir de este análisis, se plantean varias discusiones que dejan abiertas diversas preguntas para el Trabajo Social y se esbozan nuevos horizontes para la investigación y la intervención. Esta reflexión espera ser parte del camino que ha emprendido nuestra disciplina para la generación de conocimiento, de nuevas comprensiones y apuestas en torno a lo juvenil, reconociendo nuestro papel de acompañar la potencialización de 
Joya-Valbuena

capacidades y de acciones políticas transformadoras desde los escenarios cotidianos hasta aquellos que ponen en entredicho los órdenes dominantes en las estructuras sociales.

\section{Metodología}

Esta investigación documental, tuvo su origen en una inquietud que surgió en el marco de la práctica académica "Hábitat y Trabajo Social" del departamento de Trabajo Social de la Universidad Nacional de Colombia, Sede Bogotá, desarrollada por la autora durante el año 2016ํㅗ. El encuentro y la interacción con jóvenes en edad escolar (13 a 18 años) en el conjunto de Vivienda de Interés Prioritario (VIP) Plaza de la Hoja², llamó la atención del equipo de trabajo respecto a las subjetividades de los jóvenes, hombres y mujeres, y al sentido que atribuyen a sus relaciones cotidianas y a sus formas de experimentar el mundo.

Es así cómo, a partir de las reflexiones derivadas de la práctica, se planteó la necesidad de construir un cuerpo teórico-disciplinar sobre la categoría de subjetividad juvenil y en este sentido, se planteó una propuesta que tuvo como objetivo general: "desarrollar un marco de referencia teórico y conceptual sobre el sujeto joven a partir de los desarrollos del Trabajo Social Colombiano, que permita orientar la intervención con esta población". Teniendo en cuenta este horizonte, se decidió construir un estado del arte, comprendiéndolo como "una modalidad de la investigación documental que permite el estudio del conocimiento acumulado escrito dentro de un área específica" (Londoño-Palacio, Maldonado-Granados y Calderón-Villafanéz, 2014, p. 6).

Con respecto a los criterios que permitieron delimitar la revisión de literatura, se partió del carácter disciplinar de la investigación; de este modo, se revisaron las bases de datos Latindex y Publindex para identificar las revistas de Trabajo Social colombiano que se encontraban indexadas ${ }^{3}$. Se procedió con la revisión de los números virtualmente

1 La práctica "Hábitat y Trabajo Social" durante el año 2016, fue un espacio que se consolidó a través de la alianza realizada por el departamento de Trabajo Social de la Universidad Nacional de Colombia, Sede Bogotá, con la comunidad residente de Plaza de la Hoja para el trabajo con niñas y jóvenes participantes en el proceso comunitario "Club Loto de la Hoja".

2 El proyecto Plaza de la Hoja, es un conjunto residencial que cuenta con 457 unidades de vivienda que fueron entregadas a población víctima del conflicto armado en el año 2015, como parte del programa de " 100.000 viviendas gratis" que era llevado a cabo por el gobierno nacional en ese momento. Una de las particularidades más importantes de este proyecto, es que fue localizado en una zona céntrica de la ciudad como una apuesta de la administración distrital para combatir la segregación socioespacial y favorecer la interacción entre distintos sectores sociales, propendiendo también por el acceso al derecho a la ciudad de los residentes de viviendas de interés prioritario.

3 Para el año de la investigación (2017), se seleccionaron las siguientes: revista de Trabajo Social de la Universidad Nacional de Colombia; revista de Trabajo Social de la Universidad de Antioquia; Eleuthera, revista de la Universidad del Caldas; Prospectiva, revista de la Universidad del Valle; revista Tendencias y Retos de la Universidad de la Salle; revista colombiana de Trabajo Social del Consejo Nacional para la Educación en Trabajo Social (Conets), y la revista de Trabajo Social de la Universidad Pontificia Bolivariana. 
Joya-Valbuena

disponibles de cada una de ellas, para encontrar artículos que desarrollaran las categorías de sujeto/subjetividad y joven/jóvenes/juventud/adolescentes/adolescenciat ${ }^{4}$. En este primer rastreo se contó con treinta (30) textos, que fueron clasificados en tres grupos: a) relacionados directamente con la subjetividad (7), b) sistematización/investigación de experiencias organizativas y de intervención con jóvenes (7) y c) relacionados con problemas sociales asociados a la juventud (16).

Luego de una primera lectura, que tuvo como criterio fundamental que los documentos desarrollaran la reflexión sobre la subjetividad, se seleccionaron solamente veintitrés (23), dado que los siete restantes, no profundizaban sobre el asunto sino que abordaban las problemáticas juveniles desde otras ópticas.

De esta manera, se consideró pertinente poder ampliar la búsqueda para contar con un cuerpo documental más nutrido para la revisión. Se rastrearon entonces, en las mismas bases de datos (Latindex y Colciencias), revistas de Ciencias Sociales interdisciplinarias que entre sus temas trabajaran asuntos relacionados con la juventud, con el propósito de identificar artículos cuyos autores fueran trabajadores y trabajadoras sociales ${ }^{5}$. Inicialmente se contó con treinta y un (31) revistas, sin embargo, al revisarlas en detalle según las categorías y el criterio disciplinar de la investigación solamente se seleccionaron tres artículos, de tres revistas diferentes ${ }^{6}$.

Una vez se contó con los documentos que cumplían con los criterios de selección (veintiséis), se procedió con la lectura, análisis y clasificación de los mismos. Al respecto, Londoño-Palacio et al. (2014) indican que la construcción de estados del arte cuenta con dos metodologías: la heurística y la hermenéutica. La primera tiene que ver con la indagación y revisión de los documentos y la segunda con el análisis crítico que se hace al dialogar con las fuentes consultadas.

Para este caso, en lo correspondiente a la revisión (heurística), luego de la delimitación del tema y la selección de los artículos, se identificaron cuatro tendencias de trabajo: a) reflexión/investigación sobre el sujeto joven y sus relaciones cotidianas, b) experiencias organizativas juveniles y políticas públicas, c) juventud y familia y d) problemas sociales asociados a la juventud. Así mismo se logró delimitar las principales perspectivas epistemológicas desde las cuales se ha abordado el tema, entre ellas se encontraron: la

4 Si bien el horizonte de la investigación plantea una crítica a la noción de "adolescencia" se consideró pertinente revisar los desarrollos existentes en relación con esta categoría y la subjetividad.

5 En este sentido, a pesar de que se amplió la búsqueda a revistas de otras áreas, se mantuvo el criterio disciplinar con respecto a los autores. Para ello, se llevó a cabo una revisión de los perfiles, desde la información disponible sobre sus currículos académicos en línea.

6 Estas fueron Tabula Rasa, Revista Latinoamericana de Ciencias Sociales, Niñez y Juventud y la revista Nómadas. 
Joya-Valbuena

sistémica, el post-estructuralismo, el construccionismo social, la fenomenológicohermenéutica y la perspectiva crítica y decolonial ${ }^{7}$.

Ahora bien, en lo correspondiente a la fase de análisis del estado del arte (hermenéutica), se construyó una matriz que permitió desentrañar las ideas fuerza de cada texto y en la cual se registraron: a) concepción de juventud manejada por cada autor/autora b) territorios que referencian los trabajos, c) identificación del tipo de artículo (reflexión o investigación), d) marcos de referencia/enfoques, y e) principales contenidos del texto.

Con ello fue posible proceder a un momento de interpretación, análisis y categorización de los hallazgos, identificando las discusiones que permitirían desarrollar un diálogo entre autores para la construcción del referente teórico-disciplinar esperado. De esta manera, se alcanzó una saturación temática en la cual la matriz arrojó como elementos comunes: a) la construcción histórica de un discurso hegemónico sobre el/la joven, b) la existencia de un sistema de dominación adultocéntrico que se reproduce a nivel estructural y cotidiano; y c) la pertinencia de un nuevo abordaje sobre el sujeto joven desde la capacidad de agencia y la resistencia. A partir de allí y teniendo como base estos tres ejes centrales de la reflexión, se codificaron los textos de manera que fuera posible la elaboración del estado del arte cuyos hallazgos se presentan a continuación.

\section{Hallazgos}

\subsection{Revisión histórica de la concepción de juventud en Occidente y en Colombia}

El abordaje de la discusión y reflexión desde el Trabajo Social colombiano sobre el sujeto joven contemporáneo tiene como punto de partida las construcciones simbólicas y las representaciones que se han consolidado al respecto en la sociedad occidental y, particularmente, en el contexto colombiano. Para ello, algunos autores revisan cómo se ha entendido la juventud a lo largo de la historia hasta llegar a la concepción imperante de joven, teniendo en cuenta la dimensión temporal y los aspectos geopolíticos que inciden en esta construcción socio-histórica.

Amador (2013) afirma que somos una sociedad que se ha constituido a partir de la experiencia colonial y, por tanto, se conforman dentro de ella relaciones asimétricas que deshumanizan a los otros desde los discursos hegemónicos. Al respecto, Martínez-Posada y Barragán-Giraldo (2008) desarrollan una revisión arqueológica del concepto de juventud

7 Es importante señalar que las perspectivas epistemológicas aquí esbozadas, no suponen el marco teórico de la investigación dado que es un estado del arte. Sin embargo, se plantean aquí con el propósito de tener una perspectiva general sobre las principales tendencias en la producción académica del Trabajo Social respecto al tema que nos convoca. 
Joya-Valbuena

que permite analizar las transformaciones y la evolución de los discursos en la sociedad occidental desde la antigüedad hasta la contemporaneidad. Hurtado-Rodríguez (2011) por su parte, analiza en profundidad la manera como estas concepciones se han expresado y desarrollado en Colombia. Teniendo en cuenta las múltiples imbricaciones entre estas representaciones simbólicas y los impactos que han tenido en la vida cotidiana y en nuestra cultura, ponemos en diálogo estas dos perspectivas que permiten situar la reflexión en torno a la dominación etaria y a la estigmatización de la juventud:

a) Las sociedades preindustriales: según Martínez-Posada y Barragán-Giraldo (2008), durante este periodo las y los jóvenes eran considerados como sujetos que a través de rituales de transición entrarían a ser parte del sistema productivo y reproductivo para garantizar la supervivencia del grupo. En las sociedades clásicas fueron llamados "efebos" y en ellos se exaltaban las cualidades del joven griego que estaba inmerso en actividades culturales y de reflexión que resultaban fundamentales para la Polis. En la Edad Media, en cambio, no hay un reconocimiento de lo juvenil, desde la infancia se preparaban para el oficio que les correspondiera por nacimiento y no había distinción alguna con los adultos; es en el renacimiento donde la naciente burguesía empieza a configurar representaciones sobre la juventud como una edad que preparaba para las responsabilidades adultas.

Hurtado-Rodríguez (2011) recoge estos tres periodos de la historia de Occidente y los unifica en la época prehispánica en lo que concierne al contexto latinoamericano. En nuestros territorios, a la niñez y la juventud se les otorgaba un gran valor como reproductores de la cultura y la estructura social, por tanto, su crecimiento era acompañado por rituales y por la comunidad. Después de la colonización española, esta visión particular fue destituida por el modelo de civilidad europeo que buscaba formar jóvenes obedientes que reprodujeran los valores dominantes a través de diferentes dispositivos de control que variaban según el grupo social al que pertenecían ${ }^{8}$.

b) El joven del capitalismo: las sociedades industriales europeas transitaron de no diferenciar al joven del adulto hacia la construcción de un "arquetipo de juventud" (Martínez-Posada y Barragán-Giraldo, 2008, p. 358). La consolidación de la familia burguesa y de las instituciones de la modernidad situaron al joven en un lugar de preparación antes de ingresar al mercado laboral. Este arquetipo fue adoptado en las clases privilegiadas mientras que los jóvenes obreros eran concebidos como delincuentes. En el siglo XX, se da una transformación en la que las y los jóvenes se constituyen como sujeto político en un momento histórico convulsionado de manera diversa según los contextos; cuestionan el mundo adulto y el control que es ejercido por este. Sin embargo, pasado este periodo, la

8 Para los sectores privilegiados surgía la educación y los manuales, mientras que para los demás grupos sociales, la evangelización y el trabajo eran los mecanismos de dominación utilizados para cambiar los modos de ver el mundo y las concepciones sobre la infancia y la juventud (Hurtado-Rodríguez, 2011). 
sociedad post-industrial ve nacer a un joven que es percibido como conformista, desmovilizado políticamente y que realiza una serie de prácticas peligrosas para la sociedad, pero a la vez se exalta la "eterna juventud" como anhelo (Martínez-Posada y Barragán-Giraldo, 2008).

Puede verse cómo en el cambio de siglo (del XX al XXI) se da una transformación drástica entre el joven que cuestionaba el orden hacia uno desinteresado. Amador (2013) da pistas para comprender esto, señalando que las nuevas formas de control, hacia finales del siglo XX y entrado el XXI, utilizaron el discurso de la competitividad y la ganancia personal para hacer frente a estos colectivos que amenazaban el orden establecido. HurtadoRodríguez (2011) precisa que en Colombia esta tensión ha adoptado otra forma mediante una paradoja de carácter jurídico y cultural según la cual se reconoce a los niños, niñas y adolescentes ${ }^{9}$ como sujetos de derecho pero que a la vez son vistos desde su condición de problema y desde la necesidad de controlarlos.

Ahora bien, esta relación entre la consolidación de un concepto hegemónico de juventud en la sociedad occidental y en el contexto colombiano, permite ver cómo estos discursos plantean paradojas que legitiman el sistema de dominación adultocéntrico, pues reconocen a jóvenes, niños, niñas como "promesas de futuro", al tiempo que se les estigmatiza y se les regula bajo una lógica de funcionalidad y control.

\subsection{Ser joven en una sociedad adultocéntrica}

Al reconocer que las subjetividades se constituyen a partir de la participación en discursos estructurales que son apropiados por los sujetos en sus relaciones cotidianas, es fundamental abordar el sistema de dominación adultocéntrico en la reflexión sobre la juventud. Montenegro-Martínez (2004) analiza esta jerarquización desde la categoría de posiciones de sujeto, que muestra cómo al interior de los procesos históricos que configuran las sociedades se generan discursos que ponen a ciertos actores en lugares de privilegio y a otros en condiciones subalternas, lo que da paso al conflicto social y a la posibilidad de subvertir estas relaciones.

Desde esta comprensión, se reconoce la existencia del adultocentrismo como una forma de dominación que ha sido naturalizada. Ser adulto es una condición de privilegio, Amador (2013) afirma que existe "un tipo de hegemonía social que evidencia una suerte de asimetría naturalizada entre la figura adulta y las de otros individuos" (p. 143). El deber ser se construye a partir del ideal de maduración y se deben seguir modelos establecidos por quienes se considera ya han llegado a esta meta. Ser niño, niña o joven debe tener como

9 Si bien nuestra perspectiva se aparta de la noción de "adolescencia", se plantea en este apartado como parte de la crítica hecha a los instrumentos jurídicos y normativos que rigen la sociedad colombiana. 
Joya-Valbuena

proyección final llegar a ser lo que el adulto propone e introyectar las normas que le llevarán a ello.

Las instituciones y los espacios de interacción se determinan a partir de este discurso, la discriminación etaria se ha legitimado de diversas maneras que convierten en problema a los sujetos que no responden a las exigencias sociales y culturales establecidas. De este modo, se generan dinámicas estigmatizadoras hacia las y los sujetos jóvenes que van desde los discursos sobre cómo deben comportarse hasta la criminalización de sus modos de vestir y de estar en el mundo, consolidando en la cultura concepciones negativas asociadas a una "etapa".

Es así como el ejercicio del poder adulto se ha apoyado en la construcción de imaginarios sobre el sujeto joven y su lugar en la sociedad. De este modo, circulan representaciones que no se cuestionan y que atraviesan las subjetividades y las interacciones en las que las y los jóvenes están inmersos. A partir de la revisión realizada, pudieron trazarse tres concepciones dominantes que reproducen la subalternización y la discriminación etaria en distintos escenarios. Estas son: a) el joven como adulto incompleto, b) la existencia de un único ideal de juventud, y c) el joven problema o no-normatizado. Así mismo, estas concepciones atraviesan la intervención del Trabajo Social, por lo cual, desde el giro conceptual y epistemológico planteado, se considera fundamental realizar un ejercicio reflexivo sobre la profesión que se presenta como d) la intervención patologizante del Trabajo Social.

a) El joven como un adulto incompleto: este discurso legitima la subalternidad del joven desde una visión etapista que tiene su plenitud en alcanzar la adultez. Varios autores hacen una fuerte crítica a las concepciones de la y el joven como un "inmaduro" o "inmadura". En palabras de Osorio-Pérez (2005), la juventud es asumida por la sociedad como "una edad en donde no se es, sino en donde se prepara para ser" (p. 123). Desde esta perspectiva, la juventud se concibe como un momento pasajero en el que se deben apropiar los valores de la sociedad y prepararse para ingresar al mercado (Restrepo-García, 2014). El adultocentrismo señala el camino y los asuntos que deben considerarse importantes, de este modo los sentidos y experiencias juveniles que se construyan por fuera de ellos son percibidos como insignificantes.

La responsabilidad es comprendida desde lo que demanda el sistema productivo, por tanto, los proyectos de vida deben ser funcionales para el neoliberalismo y la noción de incompletud se complementa con la perspectiva de vulnerabilidad (Montenegro-Martínez, 2004; Palacio-Valencia, 2011). El joven es percibido como un ser desvalido que requiere tutoría de otros, carece de autonomía y de poder decisorio frente a sí mismo y su entorno: "son invisibilizados como actores sociales capaces de comprender, opinar y participar" (Osorio-Pérez, 2005, p. 124). 
Joya-Valbuena

b) Existencia de un único ideal de juventud: en la búsqueda de que el sujeto joven se adapte a lo demandado por la sociedad, se crea una única forma posible de estar en el mundo y de expresarse. En este modelo se establecen las formas correctas de vestir, de habitar, de hablar y de pensar; la diferencia supone una amenaza y, por consiguiente, se legitima la estigmatización y la confrontación con quienes no se ajustan a estos modos (Medina-Bermúdez, 2015).

c) El joven problema o no-normatizado: el predominio de este único modo de ser impuesto se sustenta en la reproducción y apropiación de los valores dominantes: quien no se ajusta a ellos es considerado transgresor y por tanto, peligroso. Las resistencias y búsquedas de las y los jóvenes son vistas como amenazas al orden, de allí que empiecen a circular discursos que lo convierten en problema social y por tanto, exigen un pronto ingreso a la norma. Desde esta concepción, pareciera que lo que debe ocuparnos en la comprensión y trabajo con los sujetos jóvenes es su "incapacidad" para adaptarse y responder a las demandas sociales.

Frente a esta mirada, desde la apuesta del Trabajo Social colombiano para transformar esta perspectiva, se hace una fuerte crítica a esta tendencia de convertir a las y los jóvenes en problema social. Los autores y autoras reconocen que junto con las representaciones asociadas a la delincuencia, aparecen el joven adicto, el joven violento y el joven que requiere vigilancia constante de la familia y de las instituciones (Amador, 2013; MontenegroMartínez, 2004; Palacio-Valencia, 2011).

Así mismo, estas concepciones negativas se apoyan en diversos mecanismos para reproducir esta visión, tal como el lenguaje cotidiano, las leyes y los medios de comunicación. Restrepo (2010) se refiere a estas como formas de menosprecio "que atentan contra la autonomía, el autorrespeto y la autorrealización del sujeto" (p. 182). El lenguaje cotidiano normaliza la discriminación y da lugar a los prejuicios, los chistes y la descalificación. La ley por su parte, promueve las lógicas de control y vigilancia, que a su vez persiguen las subjetividades que no se acogen al modelo de civilidad establecido (Montenegro-Martínez, 2004; Palacio-Valencia, 2011). Los medios de comunicación moldean las opiniones de las personas convirtiéndose en una herramienta fundamental para la transmisión y extensión de la estigmatización (Restrepo-García, 2014).

Este escenario de ver al y a la joven como transgresores de la norma y por tanto un problema, supone la paradoja que bien expresa Hurtado-Rodríguez (2011) entre la concepción de los "adolescentes" como sujetos de derechos pero a la vez amenazas sociales que deben controlarse mediante la ley. Esta tensión se hace también visible en la afirmación de "el joven es el futuro" pero debe cumplir la norma establecida (Martínez-Posada y Barragán-Giraldo, 2008; Palacio-Valencia, 2011). 
Joya-Valbuena

d) La intervención patologizante del Trabajo Social: Castiblanco-Lemus, SerranoPiraquive y Suárez-Cruz (2008), muestran cómo estas construcciones simbólicas y discursivas sobre la juventud, se encuentran presentes en el quehacer del Trabajo Social y en algunas perspectivas de intervención. Al respecto afirman que:

Acerca de la cuestión juvenil, Trabajo Social ha mantenido una mirada epidemiológica, $<<$ patologizante>> y por lo tanto más que la pregunta por los jóvenes, se interroga por la problemática juvenil, enfatizando en aspectos <<anormales >> asociados al hecho de ser joven (...). En consecuencia, las respuestas se han asociado a una mirada del joven como carente víctima y necesitado y en ese sentido requiere ser incluido como beneficiario de servicios institucionalizados (p.22)

Ahora bien, dentro de esta discusión sobre la funcionalidad de la intervención para la sociedad adultocéntrica, aparece también la pregunta por el lugar de las políticas sociales en el abordaje de la cuestión juvenil. La realidad paradójica de la infancia y la juventud como sujetos tutelados y protegidos por el Estado, la sociedad y la familia (Palacio-Valencia, 2011) hace que sean poblaciones objeto ${ }^{10}$ de políticas públicas. Peralta-Duque (2016) precisa que, en el contexto latinoamericano, se han consolidado tres enfoques para la política pública de juventud, asociados a los estándares planteados por organismos multilaterales como el Banco Interamericano de Desarrollo (BID), el Banco Mundial (BM) o la Comisión Económica para América Latina y el Caribe (CEPAL). En la tabla 1, puede verse cómo las concepciones de juventud que hasta aquí hemos analizado permean la mayoría de estas perspectivas y al mismo tiempo, suponen una intencionalidad que responde a las demandas de la sociedad adultocéntrica.

Tabla 1. Enfoques Políticas Públicas de Juventud.

\begin{tabular}{ccc}
\hline Tipo de enfoques & Concepción de juventud & Intencionalidad \\
\hline Tradicionales & $\begin{array}{c}\text { Etapa de transición a la } \\
\text { adultez }\end{array}$ & Preparar para el futuro \\
Reactivos & Etapa problemática & Disminuir riesgos \\
Avanzados & $\begin{array}{c}\text { Actores estratégicos del } \\
\text { desarrollo }\end{array}$ & $\begin{array}{c}\text { Fortalecer la } \\
\text { ciudadanía y la } \\
\text { producción cultural }\end{array}$ \\
\hline
\end{tabular}

Fuente: elaboración propia, con base en lo propuesto por Peralta-Duque, 2016

\footnotetext{
10 Se utiliza aquí la palabra "objeto" y no sujetos, porque a partir de los aportes de los autores y autoras, se defenderá la tesis de que la juventud es receptora pasiva de programas no participativos.
} 
Joya-Valbuena

Puede verse entonces, que gran parte de las políticas se sustentan en la visión de incompletud, inmadurez, vulnerabilidad e incluso de peligrosidad de las y los jóvenes. Por la misma vía, Peralta-Duque (2016) analiza que, en distintos escenarios de participación, enmarcados en los enfoques denominados "avanzados", se centralizan las decisiones y las y los jóvenes se ven restringidos por las dinámicas institucionales que los relegan a la mera asistencia a espacios en los que no tienen ninguna incidencia. Esto a su vez, se relaciona con lo que expresa Amador (2013) cuando afirma que las instituciones en su relación con las y los jóvenes buscan "convertirlos en cifras de la política pública que los interviene; reeducarlos para que no le hagan daño a la sociedad" (p. 143). La tensión derechos-control se convierte en una instrumentalización de las y los sujetos a través de la política pública que se acoge estas concepciones negativas y por tanto asume enfoques reactivos. Al respecto Castiblanco-Lemus et al. (2008) afirman que: “Los sujetos de las políticas públicas deben encajar en una categoría: delincuente, drogadicto, violento, desordenado sexualmente, o por el contrario, indefenso, víctima, proclive a enfermarse, a ser excluido" (p. 20).

Con estas acciones se invisibilizan y se callan las opiniones e iniciativas juveniles y se busca intensificar el control desde la ley que re-educa (Álvarez-Franco y Zapata-Posada, 2013; Castiblanco-Lemus, 2005); no se cuestionan las condiciones estructurales económicas, sociales y culturales sino a individuos considerados "desviados". En este sentido, no puede entenderse la política pública desligada de una estructura y de un modelo que busca reproducirse, por lo que es importante también enmarcar el análisis en un sistema económico/social marcado por un ideario particular.

\subsection{Adaptación, estigmatización y neoliberalismo}

De acuerdo con López-García (2011), la subjetividad juvenil se construye dentro del modelo en el que están insertos, que busca intensificar la identificación y aceptación del mismo. Las y los jóvenes se convierten en blanco del neoliberalismo para su reproducción que también está apoyada en la homogenización y en los estilos de vida únicos. Las pautas culturales, sociales y económicas exigen a todos los sujetos incorporar el discurso de la competitividad y de la exaltación de lo individual; los intereses y proyectos de vida deben estar orientados y diseñados con base en la inserción al mercado y la posibilidad de consumir. La noción de "completud" está vinculada a hacer parte del sistema productivo y a una serie de construcciones simbólicas que lo legitiman y perpetúan.

Esta mercantilización del sujeto joven lo ha convertido en el consumidor por excelencia, las búsquedas que se emprenden a nivel identitario son aprovechadas por el mercado para que, a través de las marcas, los objetos y las imágenes se conviertan en elementos claves para los grandes productores, lo que genera otro tipo de tensiones vinculadas con el poder adquisitivo y la clase social de quienes son blanco de la publicidad y los medios. Así, la condición juvenil entra en la encrucijada del ser-tener que disminuye 
Joya-Valbuena

otras posibilidades de ser sujeto; es necesaria la adscripción e inserción, el asumirse como incompletos y como individuos que trazan un camino determinado para hacer parte del orden (López-García, 2011).

Ahora bien, entre las estrategias neoliberales para controlar, soslayar y adaptar al sujeto joven, surge una nueva paradoja, relacionada con la tensión entre la discriminación/estigmatización y el anhelo de la "eterna juventud". A pesar de las construcciones simbólicas negativas, circula y se internaliza el deseo de permanecer joven, lo cual relativiza esta categoría invisibilizando la subalternización que subyace a la jerarquía intergeneracional. (Martínez-Posada y Barragán-Giraldo (2008) lo nombran como el "culto a lo joven" (p. 360) que exalta la posibilidad de mantener una apariencia y un cuerpo joven. Sin embargo, no es cualquier cuerpo sino el estereotipado por los medios y por las industrias; es la "idea de juventud y belleza que realza unos valores corporales enmarcados en una producción económica y social” (Montenegro-Martínez, 2004, p. 131).

Teniendo esto como base de la reflexión sobre esta paradoja, es importante no confundir el ideal de ser joven con la subjetividad juvenil, pues el primero está enmarcado en el consumo y en la propagación de un imaginario con intereses mercantiles, mientras que la segunda nos sitúa frente a unos sujetos con una posición desigual en un entramado de relaciones de poder y de estructuras de dominación.

\subsection{El "deber ser" del joven y la familia}

Además de la relación adultocentrismo-neoliberalismo, también logró identificarse en la literatura consultada una lectura crítica del lugar de la familia al interior de este sistema de dominación. Los autores y autoras presentan cómo la sociedad neoliberal oculta su responsabilidad en las complejidades que atraviesan las y los jóvenes y cómo esta negación está acompañada de una demanda a la familia de servir como instrumento que forme a sus miembros para reproducir el orden. La visión de vulnerabilidad y la paradoja derechoscontrol, supone que la familia tenga "la responsabilidad primordial de la crianza" (PalacioValencia, 2011, p. 20) que tiene que acogerse a los parámetros sociales y culturales. Por esta razón, ante distintas situaciones, se señala a las familias como responsables de crear individuos amenazantes y riesgosos para la sociedad; Palacio-Valencia (2011) muestra cómo en los casos de jóvenes penalizados, la criminalización se extiende hacia los sujetos adultos vinculados consanguíneamente al o a la joven.

En este punto, es importante aclarar que lo problemático no es que la familia se constituya como un espacio primordial para la construcción subjetiva, sino que se le exige que lo haga de acuerdo a unos valores dominantes. Además de ello la sociedad adultocéntrica y neoliberal se exime a sí misma de su corresponsabilidad en la construcción de las y los sujetos jóvenes y en la generación de diversos problemas sociales que les afectan. 
Joya-Valbuena

Es así como este mandato a la familia está condicionado por la hegemonía de la familia nuclear que co-reside y ampara económicamente a las y los jóvenes (Palacio-Valencia, 2011). No se tiene en cuenta la diversidad familiar presente en el contexto y por tanto las transgresiones se justifican en el hecho de que las y los jóvenes no crezcan dentro de la tipología deseada y aceptada socialmente. Así mismo, la insistencia de este discurso también tiene implicaciones en las construcciones subjetivas; López-Castillo (2009), señala que entre las y los jóvenes "existe una añoranza" (p. 372) de la familia nuclear ideal, en contextos en los que se presentan otros tipos de organización familiar y en los que los sujetos han sido discriminados por no enmarcarse en este imaginario.

Por otra parte, el lugar de la familia se complejiza más cuando esta reproduce la lógica asimétrica intergeneracional del adultocentrismo. La perspectiva que ve al joven y a la joven como un ser incapaz de autonomía, se introyecta en quienes jurídicamente tienen su tutoría, generando una multiplicidad de tensiones que devienen en conflictos de alta intensidad. Las y los jóvenes por su parte, construyen representaciones sobre sí mismos a partir de un lugar de subalternidad en sus familias (Alvear-Romero y Herazo-Dilson, 2006), se naturaliza una mirada en la que debe obedecerse a las personas adultas sin importar el tipo de mandato y en la que no se tiene la capacidad de tomar decisiones autónomamente.

Esta visión está acompañada, además, por las expectativas construidas al interior de la familia sobre las y los jóvenes. Las personas adultas esperan que quien está bajo su cuidado se comporte como ellos esperan y conforme un proyecto de vida acorde con sus valores (Castiblanco-Castro y Lora de las Salas, 2008), lo cual genera fuertes tensiones en las relaciones familiares cuando las y los jóvenes no se sienten identificados con estas búsquedas. De este modo, la discriminación también tiene lugar entre los más próximos pues se considera que el rechazo de las y los jóvenes a la autoridad y a los ideales de los adultos no tienen trascendencia y son solo actos de rebeldía que deben ser coartados (Restrepo, 2010).

Ahora bien, este último punto implica pensar que la complejidad de nuestro contexto no solo está relacionada con lo que atañe directamente a lo familiar, sino que se amplía a otros escenarios y condiciones estructurales que deben ser tenidas en cuenta para pensar cómo se construye la subjetividad de las y los jóvenes. Si bien existe un marco general que permite ver cómo se han construido ciertos imaginarios, este debe leerse cruzando las categorías de clase, territorio ${ }^{11}$, género, etnia entre otras, que demarcan la diversidad de las y los jóvenes y es a partir de allí que se plantea una transformación en la mirada patologizante y homogenizante de una "etapa" anclada a la falta de autonomía, hacia una

11 Es importante llamar la atención sobre la multiplicidad de contextos geográficos y espaciales, pues las diversidades expresadas tanto en lo urbano como en lo rural y en los territorios de borde implican abordajes heterogéneos que conlleven una lectura cuidadosa de los contextos con los que nos relacionamos como trabajadores y trabajadoras sociales. 
Joya-Valbuena

noción transformadora que reconoce en las y los jóvenes sujetos capaces de agenciar su propia realidad y sus contextos desde el reconocimiento como actores sociales y políticos.

\subsection{Nuevos abordajes del joven: del sujeto problemático al sujeto con agencia}

Una vez reconstruidos los aportes con respecto a la construcción cultural e histórica de una sociedad que subalterniza a las y los jóvenes, se plantea la apuesta del Trabajo Social colombiano por comprender lo juvenil desde la subjetividad, ya no desde la visión dominante que problematiza a los individuos, sino desde una perspectiva de agencia. Se responde entonces a la invitación hecha por Castiblanco et al. (2008) en tanto sugieren que el Trabajo Social haga un desplazamiento conceptual en lo que se refiere a su concepción e intervención con las y los jóvenes.

Abordar la condición juvenil desde la subjetividad y la identidad, implica situarse en un entramado de relaciones que las construyen, a su vez que se reconoce la capacidad de las y los jóvenes para tomar decisiones y opciones. Referirnos a la identidad no se reduce a la adscripción a cierto colectivo o grupo cultural de manera estática y uniforme en la que los individuos apropian marcos simbólicos de manera lineal (Montenegro-Martínez, 2004); sino que sitúa a los sujetos en un contexto histórico y cultural en el cual construyen una trama de vida en relación con los otros y otras (Castiblanco et al., 2008). De esta manera, la construcción identitaria y subjetiva es un proceso constante, en el que cada uno y cada una va entretejiendo una manera de narrarse a sí mismo desde la significación de sus vivencias y encuentros (Cifuentes-Patiño, 2008) ${ }^{12}$.

Por otra parte, López-García (2011) y Torres-Victoria (2013) precisan que no existe la subjetividad en singular, sino que, a partir de las tensiones y contradicciones de las relaciones sociales, un sujeto se constituye a partir de múltiples subjetividades y distintas formas de expresarla. En este sentido, se reconoce la diversidad de experiencias y de interacciones como parte fundamental de nuestro enfoque y así mismo, se defiende la idea de que no es posible conocer a un sujeto de manera unívoca abordando solamente una dimensión, sino que cada persona teje su experiencia desde la complejidad de sus relaciones y de la construcción de sentidos que va elaborando a lo largo de su trayectoria como un

12 Es importante precisar que el abordaje de las categorías de identidad y subjetividad contiene en sí mismo múltiples entradas, pues en las Ciencias Sociales se encuentran desarrollos desde distintas perspectivas epistemológicas. Consideramos pertinente mantener desde nuestra profesión-disciplina una mirada flexible y abierta que permita identificar la pertinencia de cada uno de estos paradigmas tanto en la intervención como en la investigación, siguiendo la invitación que hace Zemelman (2011) de aproximarnos a las categorías como "posibilidades de contenido" (p. 218) que no se cierran a una única "definición" sino que se van transformando a partir de las exigencias que la realidad social plantea para la construcción de conocimiento. 
proceso inacabado y en constante transformación (Jiménez-Gómez y Ramírez-Loaiza, 2016; Ortiz-Ruiz, 2012).

Es así como precisamos que el encuentro del Trabajo Social con las y los jóvenes requiere de una aproximación cuidosa, en la cual, se observen las particularidades de cada contexto y se reconozcan las subjetividades con las que entramos en diálogo, sin precisar el uso de "recetas", modelos de intervención o metodologías de investigación "pre-diseñadas" que responden a las lógicas del adultocentrismo y el "deber ser" de la sociedad occidental.

Ahora bien, un elemento clave en la comprensión de las subjetividades juveniles tiene que ver con el análisis sobre la importancia del encuentro con otros y otras jóvenes en la construcción identitaria. Si bien el encuentro con la alteridad se da en diversos escenarios (familia, escuela, sociedad, etc.), la interacción con quienes se identifican dentro de la misma generación es una dimensión a la que se le atribuye mayor importancia y sentido por parte de las y los jóvenes.

\subsection{El encuentro intra-generacional como parte de la construcción subjetiva}

La generación es referida por Montenegro-Martínez (2004) como una categoría que “acompaña a las personas durante toda su vida" (p. 127) pues a pesar de pasar de una edad a otra, han nacido y crecido en un momento histórico específico, aunado a las dinámicas propias de los contextos y territorios. A partir de la revisión de la literatura consultada, puede afirmarse que desde el Trabajo Social colombiano se ha evidenciado que la relevancia de este encuentro intrageneracional tiene que ver con la identificación y con los vínculos que se construyen, los cuales no se escapan de distintas tensiones y conflictos que pueden emerger en estas interacciones.

Las y los jóvenes no establecen relaciones de amistad con todos los sujetos de su generación; existen algunos factores que influyen en que se dé un mayor acercamiento y la construcción de un lazo más fuerte con algunos que con otros. El encontrar personas que comparten algunos elementos del pasado y de lo que viven en el presente, genera sentimientos de empatía y de identificación que hacen que los y las jóvenes perciban que no son los únicos que atraviesan ciertas situaciones, sino que se fortalecen a partir del reconocimiento, además de establecer dinámicas de cooperación, confianza y ayuda desde la vivencia de experiencias similares (Restrepo, 2010).

Esta identificación lleva a que los sujetos jóvenes vean en las relaciones de amistad la posibilidad de establecer una interacción simétrica basada en la confianza y en la posibilidad de compartir las tensiones que viven en su cotidianidad. El tiempo compartido genera afectividades y altas valoraciones de lo que son los otros y de lo que se vive (Ortiz-Ruiz, 
Joya-Valbuena

2012). Así mismo, se tejen relaciones horizontales en las que, contrario a otros escenarios en los que se reproduce el adultocentrismo, las y los jóvenes encuentran una mayor posibilidad de autonomía y libertad de expresión (Álvarez-Franco y Zapata-Posada, 2013; PalacioValencia, 2011; Restrepo, 2010). Ahora bien, la intensidad de los vínculos y el tiempo compartido con los amigos y amigas, hace que las y los jóvenes también entren en conflicto entre ellos en determinadas circunstancias o de manera constante (Medina-Bermúdez, 2015). Dada la importancia otorgada a la amistad, se pueden generar diversos malestares; sin embargo, esta situación puede leerse como una oportunidad para potenciar capacidades de resolución de conflictos y de diálogo con la diferencia.

Por otra parte, junto con estas relaciones al interior de la misma generación, aparecen también confrontaciones con otros sujetos que, como ya pudo verse, reproducen las lógicas del adultocentrismo en distintos escenarios cotidianos. Uno de los imaginarios más extendidos en nuestras sociedades, tanto en la relación con el joven de manera singular como con los colectivos o grupos de jóvenes tiene que ver con la "rebeldía". A partir del giro conceptual que aquí se ha planteado, se propone resignificar este discurso desde el reconocimiento de las búsquedas de autonomía que las y los jóvenes desarrollan en su vida cotidiana como parte de su posibilidad de agenciar su propia realidad (Castiblanco-Lemus, 2005).

\subsection{Resignificar la rebeldía desde las prácticas de resistencia y la búsqueda de autonomía}

La apuesta por cambiar la noción de "jóvenes rebeldes" por la de jóvenes que buscan su autonomía, se ancla en la importancia de la categoría de resistencia al interior de la comprensión de la subjetividad juvenil; una resistencia que se expresa tanto en la familia como frente a las instituciones educativas y a la sociedad que les homogeniza, individualiza y mercantiliza.

Castiblanco-Lemus (2005) hace una aproximación a los desarrollos sobre esta categoría y señala cuatro procesos que le subyacen: “poder, hegemonía, subordinación y dominación" (p. 259). La reflexión sobre lo hegemónico surge desde el pensamiento Gramsciano que opone lo subalterno a lo hegemónico (García Canclini, 1984, citado por Castiblanco-Lemus, 2005); la contradicción social y cultural caracterizada por la dominación, no es una relación polarizada, sino que hay una "interacción ideológica" (Castiblanco-Lemus, 2005, p. 259), en la que los oprimidos tienen la capacidad de resistir a las estructuras sociales, económicas y culturales. Así, la resistencia surge como modo de existencia creativo, no solo se subvierte el orden desde la confrontación directa, sino que el aparecer diferente frente a una sociedad que impone unos modelos se convierte en una forma singular y particular de resistencia. 
Joya-Valbuena

Esta idea también es soportada por López-García (2011) al señalar que las prácticas de resistencia pueden expresarse "a partir de la producción de espacios y subjetividades alternas, estratégicas, soportadas en formas de fuga que no niegan la realidad, sino que la resignifican" (p. 18). No puede entenderse la resistencia sin su relación con la identidad, la subjetividad y con el ejercicio del poder cotidiano y naturalizado ya que está estrechamente relacionada con la búsqueda de autonomía y de opciones del sujeto para gobernarse a sí mismo.

A partir de esta comprensión sobre la resistencia, que se expresa de formas distintas y que se relaciona también con la condición subjetiva, se plantean diversos escenarios y maneras en que las y los jóvenes resisten a las dinámicas de poder que los inferiorizan y coartan; entre ellos se encuentran la familia, la escuela, las estéticas que confrontan y el cuestionamiento expreso al modelo económico y político imperante.

En lo que respecta a la familia y a la escuela, puede afirmarse que existe una tendencia a concebir las relaciones entre adultos y jóvenes como problemáticas, ambivalentes y contradictorias (Alvear-Romero y Herazo-Dilson, 2006; Castiblanco et al., 2008). Esta comprensión tiende a ubicarlo como un asunto "normal" de este momento de la vida desde la visión de la juventud como "etapa" de transición y bajo la lógica de la familia normatizadora, además de legitimarse con la consigna de que son "cosas que se quitan con el paso de los años" (Restrepo, 2010, p. 185).

Es así, como abordar estas realidades desde la categoría de resistencia y no desde la "rebeldía" o "desobediencia", llama la atención sobre la importancia de resignificar y transformar los "roles" asignados tradicionalmente a instituciones como la familia y la escuela, para avanzar a la construcción de relaciones basadas en el diálogo y el reconocimiento de las perspectivas de las y los jóvenes en la toma de decisiones.

Ahora bien, en lo que respecta a los cuestionamientos a una sociedad que impone una única forma "correcta" de ser y estar en el mundo, las y los jóvenes encuentran diversas maneras de resistir a la uniformidad y universalización que plantean los discursos dominantes, a su vez que rechazan la discriminación que la sociedad adultocéntrica ha legitimado (Montenegro-Martínez, 2004; Restrepo-García 2014). Estos modos de resistir apuntan a búsquedas por ser diferentes y poder "vivenciar el carácter plural de la juventud" (Ortiz-Ruiz, 2012, p. 128). Las investigaciones realizadas por algunos trabajadores y trabajadoras sociales muestran que estas resistencias no solo son visibles mediante acciones políticas y organizativas, sino también desde las estéticas y los modos de vestir el cuerpo y de las opciones que cada uno de los y las jóvenes hace respecto a sus preferencias. Sin embargo, estas decisiones no escapan de la criminalización y la estigmatización social y cultural (Medina-Bermúdez, 2015). 
Joya-Valbuena

Por otra parte, muchas de las acciones a través de las cuales las y los jóvenes resisten, se ubican en una postura crítica frente a un modelo que exalta los valores del individualismo, la competencia y el consumo (López-García, 2011). Al respecto, Castiblanco-Lemus (2005) y Castiblanco et al. (2008) plantean que el asunto de la resistencia no puede reducirse a acciones aisladas de algunos jóvenes que no "se acomodan", sino que se convierten en acciones transformadoras que interpelan los valores dominantes y proponen otras realidades posibles.

Así, la resistencia no solamente se concibe como una alteración del orden, sino como una expresión de las transformaciones contemporáneas (López-García, 2011) que exige un cambio en las lógicas dominantes. En otras palabras, las prácticas realizadas por las y los jóvenes y sus modos de vida que generan molestias en la estructura adultocéntrica y mercantilizada, marcan derroteros para pensar nuevas posibilidades de relacionarse en la familia, en las instituciones educativas y en la sociedad en su conjunto.

\section{Conclusiones}

La aproximación a la juventud desde la categoría de subjetividad implica una mayor complejidad que la noción individualizada y homogenizadora de etapa. Los hallazgos aquí presentados dan cuenta del esfuerzo que nuestra profesión-disciplina ha hecho por construir un nuevo marco de referencia en nuestra comprensión sobre las y los jóvenes. Transitamos hacia una intervención reflexiva y a procesos de investigación que tengan en cuenta las construcciones históricas de imaginarios sociales y por tanto, que busquen hacer visibles las relaciones de dominación material y simbólica que subyacen a las situaciones donde la acción de nuestra profesión tiene lugar. Volver la mirada al sujeto, su historia, sus relaciones y su potencialidad nos lleva a pensar y repensar la manera en que acompañamos y trabajamos con las subjetividades, a su vez que la forma como las concebimos y nos relacionamos con ellas.

De este modo, el contar con un cuerpo teórico y reflexivo como el que aquí se ha desarrollado, no puede quedarse en una simple aplicación instrumentalizada de herramientas conceptuales en escenarios concretos; sino que nos invita a situarnos profesional y disciplinarmente y a cuestionar nuestras prácticas y epistemologías. En este sentido, podría pensarse de manera general que existen tres formas posibles de aproximarnos a la juventud desde el Trabajo Social: 1) la vulnerabilidad del y de la joven, 2) la necesidad de "resocializar" a un o a una joven "problemática", o 3) una mirada transformadora que acompaña a los sujetos desde sus potencialidades y su capacidad de agencia.

Ahora bien, este asunto no solamente concierne a la manera en que nos situamos frente a las subjetividades juveniles, sino que interpela a la intervención misma. Al respecto, 
Joya-Valbuena

Foucault (2001) señala que el Trabajo Social aparece como una profesión que sirve a las estructuras de poder, en cuanto cumple con una "función de vigilancia-corrección" (p. 18); esta fuerte afirmación nos llama a revisar la intencionalidad de nuestra intervención y la forma en que nos relacionamos con las y los sujetos y con las instituciones.

¿Estamos sirviendo desde el Trabajo Social a una sociedad adultocéntrica, neoliberal que busca homogenizar a las y los jóvenes y criminalizar las prácticas de resistencia? $\mathrm{O}$ tal vez ¿nuestra relación con esta población está basada en la búsqueda de obediencia y en una lógica de dominación etaria? Vale la pena hacerse estas preguntas, entre muchas otras, y ver qué tanto el adultocentrismo ha permeado nuestro ejercicio profesional y nuestras búsquedas.

Si nos situamos en las reflexiones que aquí se han desarrollado, podríamos responder a estas preguntas diciendo que nuestra profesión-disciplina ha dado importantes pasos en la transformación de la mirada y en la relación que se teje con las subjetividades juveniles. Contar con producción académica que revisa críticamente los discursos y las maneras en que estos se expresan en la vida cotidiana y en las realidades concretas, muestra que no estamos enmarcados en las lógicas funcionales de las que se nos acusa.

Sin embargo, esta invitación no se agota en las conclusiones que puedan provenir de este texto, sino que lleva a pensar que el asumir una perspectiva transformadora de las realidades de injusticia y opresión, tanto materiales como simbólicas, pasa por cuestionar nuestras propias concepciones y las formas en las que hemos introyectado los valores dominantes. El Trabajo Social no está por fuera de las disputas ideológicas y epistemológicas que circulan en nuestras sociedades y, por tanto, requiere de una constante revisión crítica de su acción.

Así mismo, quedan abiertos múltiples caminos para la reflexión y la investigación, teniendo en cuenta la diversidad de escenarios en los que el Trabajo Social puede incidir tanto desde el acompañamiento a procesos comunitarios e institucionales como en la construcción de conocimiento. Entre los principales retos que podrían abordarse, aparece el lugar de las instituciones y de la intervención profesional dentro de las mismas, allí se hace necesario el cuestionamiento a los procesos de "resocialización" y a las dinámicas relacionadas con la vigilancia y el control alrededor de las subjetividades juveniles.

Así mismo, aparece la importancia de los procesos comunitarios y del reconocimiento de las subjetividad-es en plural, desde el ejercicio de un Trabajo Social que observa, respeta "el alma del lugar" (Lederach, 2008, p. 169) y no se sitúa desde una posición mesiánica o "re-educadora", sino que entra en diálogos y en construcciones conjuntas con las y los sujetos de la intervención/investigación como actores sociales, capaces de agenciar su propia realidad y transformar situaciones de opresión. Ahora bien, en este proceso aparece 
Joya-Valbuena

como elemento central el reconocimiento de la pluralidad de contextos, escenarios y sujetos, no se habla de la subjetividad juvenil como única y homogénea, sino que emergen de manera constante nuevas posibilidades y prácticas de resistencia en lo cotidiano.

Finalmente, un asunto que no puede dejarse pasar por alto, tiene que ver con la relación juventud-familia-Trabajo Social. Siendo la intervención/investigación en familia, uno de los principales campos de nuestra identidad profesional y disciplinar, es fundamental repensar la manera como se abordan las relaciones intergeneracionales y nuestro acompañamiento desde un enfoque que escapa a las lógicas que implantan la "funcionalidad/disfuncionalidad". A lo largo del texto pudo identificarse que hegemónicamente se ha otorgado a la familia una "función" de agente socializador de las normas sociales, reproduciendo el adultocentrismo y excluyendo a la sociedad y a la cultura de su corresponsabilidad en la constitución de sujetos.

Ante este panorama, el papel del Trabajo Social es central para continuar avanzando en un ejercicio de construcción de conocimiento en el que se sigan revisando de manera crítica los valores dominantes y en el que se continúe avanzando en el reconocimiento de la multiplicidad de formas de organización familiar y de las dinámicas que se experimentan al interior de las familias en el contexto colombiano. Vale la pena profundizar en las investigaciones que en nuestro país se han desarrollado en el campo de los Estudios de Familia para tejer puentes con la reflexión sobre la subjetividad juvenil y la superación de lógicas adultocéntricas.

Quedan entonces planteadas estas preguntas y oportunidades de reflexión para nuestra profesión-disciplina en lo referente a la subjetividad juvenil, la intervención en diversos escenarios y las epistemologías que determinan nuestra acción y pensamiento. Así mismo, se abre la invitación a revisar nuestro ser y quehacer no solo en lo concerniente a la juventud, sino también en lo que tiene que ver con nuestro lugar y posturas profesionales y subjetivas en los sistemas de dominación que atraviesan las relaciones sociales.

\section{Agradecimientos}

A la profesora Esperanza Cifuentes Arcila, quien dirigió el trabajo de investigación del cual se deriva el presente artículo y quien, a lo largo de varios años, ha acompañado mis búsquedas respecto a la subjetividad juvenil y el Trabajo Social

\section{Referencias bibliográficas}

Álvarez-Franco, B., y Zapata-Posada, J. (2013). La paradoja de los afectos: tensiones entre las realidades y los deseos de jóvenes en la comuna 13 de la ciudad de Medellín- 
Joya-Valbuena

Colombia. Revista de la Facultad de Trabajo Social, 29(29), 261-294. Recuperado de https:/ / revistas.upb.edu.co/index.php/trabajosocial/article/view/2479.

Alvear-Romero, C., y Herazo-Dilson, E. (2006). Los (as) adolescentes hablan de paternidad y maternidad en Cartagena de Indias: Casos Santa Lucía y Ceballos. Revista Palobra, $7(7), 92-107$.

Amador, J. C. (2013). Condición juvenil en sociedades adultocéntricas. Revista Tendencias y Retos, 18(2), 141-156.

Cajiao, F. (2008). La adolescencia en el universo de las edades de la vida. En C. E. PinzónCastaño, R. Suárez-Prieto y G. Garay-Ariza (Eds.), Para cartografiar la diversidad de l@s jóvenes (375-413). Bogotá: Universidad Nacional de Colombia.

Castiblanco-Castro, J., y Lora de las Salas, M. (2008). Potenciación de pautas parentales resilientes de familias con hijos adolescentes. Tendencias y Retos, (13), 39-54.

Castiblanco-Lemus, G. (2005). Rap y prácticas de resistencia: una forma de ser joven. Reflexiones preliminares a partir de la interacción con algunas agrupaciones bogotanas. Tabula Rasa, (3), 253-270.

Castiblanco-Lemus, G., Serrano-Piraquive, M., y Suárez-Cruz, A. (2008). Culturas juveniles y Trabajo Social con jóvenes. Tabula Rasa, (9), 13-26. Recuperado de https:/ / revistas.unicolmayor.edu.co/index.php/tabularasa/article/view/1505.

Cifuentes-Patiño, M. R. (2008). El Sí y el Otro en la constitución de la identidad: niñas, niños y adolescentes desvinculados del conflicto armado. Revista Trabajo Social, (10), 9-27. Recuperado de https://revistas.unal.edu.co/index.php/tsocial/article/view/14073. Foucault, M. (1988). El sujeto y el poder. Revista Mexicana de Sociología, 50(3), 3-20. Recuperado de https://www.jstor.org/stable/3540551?seq=1\#page_scan_tab_contents.

Foucault, M. (2001). Trabajo Social, control social y normalización: mesa redonda de discusión con Michel Foucault. En A. Chambon, A. Irving y L. Epstein (Eds.), Foucault y el Trabajo Social. Granada: Editorial Maristán.

Hurtado-Rodríguez, H. (2011). Infancia y Adolescencia: tensiones históricas entre Estado y Sociedad. Revista Trabajo Social, (12), 119-135. Recuperado de https://revistas.udea.edu.co/index.php/revistraso/article/view/24084.

Jiménez-Gómez, K., y Ramírez-Loaiza, V. (2016). Aproximaciones a la subjetividad y socialización política de jóvenes excombatientes del conflicto armado en Colombia. Revista Trabajo Social, (18), 77-90. Recuperado de https:/ / revistas.unal.edu.co/index.php/tsocial/article/view/58573/56282.

Lederach, J. P. (2008). La imaginación moral: el arte y el alma de construir la paz. Bogotá: Grupo Editorial Norma.

Londoño-Palacio, O., Maldonado-granados, L., y Calderón-Villafanéz, L. (2014). Guía para construir estados del arte. Bogotá. International Corporation of Networks of Knowledge.

López-Castillo, C. L. (2009). Parches juveniles e imaginarios de la violencia en la ciudad de Cali. Prospectiva. Revista de Trabajo Social e intervención social, (14), 355-385. doi: 10.25100/prts.v0i14.1100. 
Joya-Valbuena

López-García, M. E. (2011). Producción y expresión de la subjetividad en la juventud contemporánea. Revista de la Facultad de Trabajo Social, 27(27), 12-21. Recuperado de https:/ / revistas.upb.edu.co/index.php/trabajosocial/article/view/2531/2297.

Martínez-Posada, J. E., y Barragan-Giraldo, D. F. (2008). Juventud y multitud: aproximaciones para abordar los movimientos juveniles. Tabula Rasa, (9), 353-38.

Medina-Bermúdez, Y. (2015). Democratización de acciones políticas a través de las prácticas artísticas realizadas por jóvenes. Eleuthera, 13, 46-63. doi: 10.17151/eleu.2015.13.4.

Montenegro-Martínez, L. (2004). Culturas juveniles y «redes generizadas». Hacia una nueva perspectiva analítica sobre la contemporaneidad juvenil en Colombia. Tabula Rasa, (2), 111-143.

Ortiz-Ruiz, N. (2012). Producción de sentidos en jóvenes de organizaciones juveniles del municipio de Santiago de Cali, Colombia. Prospectiva. Revista de Trabajo Social e intervención social, (17), 115-140. doi: 10.25100/ prts.v0i17.1146.

Osorio-Pérez, F. E. (2005). Jóvenes rurales y acción colectiva en Colombia. Nómadas, (23), 12131.

Palacio-Valencia, M. C. (2011). La delincuencia juvenil: un reto para descifrar una metáfora relacional. Eleuthera, 5, 15-35.

Peralta-Duque, B (2016). La participación juvenil en la Política Pública de Juventud, 19972011 (Caldas, Colombia). Revista Latinoamericana de Ciencias Sociales, Niñez y Juventud, $14(2), 1249-1272$.

Restrepo, A. (2010). Los jóvenes y sus luchas por el reconocimiento. Nómadas, (32), 179-193.

Restrepo-García, F. E. (2014). Arte y comunicación alternativa: una apuesta por democratizar la opinión pública desde sectores juveniles marginados. Eleuthera, 11, 163-186.

Sierra-Pardo, C. P. (2015). La irrupción del imaginario social, las subjetividades y los sujetos en las ciencias sociales como asunto relevante para la investigación en educación. Revista Trabajo Social, (17), 115-128. Recuperado de https:/ / revistas.unal.edu.co/index.php/tsocial/article/view/54768/54178.

Torres-Victoria, L. P. (2013). Organizaciones juveniles: por el camino de las identidades políticas. Eleuthera, 9(2), 156-185.

Zemelman, H. (2011). Configuraciones críticas. Pensar epistémico sobre la realidad. México: CREFAL; Siglo Veintiuno Editores. 


\section{OTROS ARTÍCULOS DE PROSPECTIVA No. 32 DE 2021}

\section{EDITORIAL}

Compromisos, dilemas y desafíos del Trabajo Social con dimensión colectiva en tiempos de pandemia

Enrique Pastor-Seller

\section{ARTÍCULOS}

La narrativa biográfica como alternativa para la comprensión del Conflicto y la violencia en Colombia: una experiencia pedagógica universitaria

Sara Sofía Castaño-Barco y Claudia BermúdezPeña

Experiencias de transición a la vida adulta de jóvenes que migraron solos. Un estudio en Aragón (España) Daniel Jiménez-Franco; Raquel Berzosa-Callén y Chabier Gimeno-Monterde

Fundamentals of Education in Intervention with Young Offenders. A Critical Review of a 'Successful' Case in Andalucía, España

David Herrera-Pastor

Subjetividad juvenil: lecturas desde y para el Trabajo Social en Colombia

Daniela Joya-Valbuena

Participación politica de los jóvenes del Valle del Cauca, Colombia, en la toma de decisiones públicas

Luis Eduardo Gutiérrez-Rojas y Amanda Ramírez-Giraldo

Victimización y perpetración de violencia en pareja adolescente y redes de apoyo en Colombia. Análisis con perspectiva de género

Johanna Alexandra Reina-Barreto
Apoyo social percibido, autoestima y maternidad adolescente: entre el respeto y la intrusión. Estudio en Traiguén, Chile

Gloria Mora-Guerrero, Luisa Escárate-Colín, Carol Espinoza-Lerdón y Andrea Peña-Paredes

El desarrollo en el Trabajo Social de Colombia: un campo transversal, disperso y polivalente

Maira Judith Contreras-Santos

Análisis de la situación laboral de las alimentadoras en la caficultura de la zona central colombiana, a la luz de la teoría de Nancy Fraser

Pablo Andrés Arango-Giraldo

Prioridades de investigación en la Obra social de empleados públicos, Mendoza, Argentina. Un estudio Delphi sobre producción de conocimientos en la seguridad social

Cecilia Amalia Molina

Producción de conocimiento y toma de decisiones. Relaciones entre academia y política pública para las familias en Colombia

José Raúl-Ruíz y Sandro Leonardo MunévarVargas

El staff de prácticas profesionales en Trabajo Social: espacio de fomento del aprendizaje autodirigido. Un estudio de caso en la Universidad Católica Luis Amigó, Colombia

Cristian Eduardo Blanco-García

\section{ARTISTA INVITADO}

Margie Reinel-Aguilar Ao Cicadidae

Prospectiva

\section{PROSPECTIVA Revista de Trabajo Social e Intervención Social}

No. 32 • jul.-dic. 2021

e-ISSN: 2389-993X • Universidad del Valle 